This is the author's final, peer-reviewed manuscript as accepted for publication. The publisher-formatted version may be available through the publisher's web site or your institution's library.

\title{
A test of the automaticity assumption of compliance tactics: discouraging undergraduate binge drinking by appealing to consistency and reciprocity
}

Amy E. Conner, Megan M. Miller, Laura A. Brannon

\section{How to cite this manuscript}

If you make reference to this version of the manuscript, use the following information:

Conner, A. E., Miller, M. M., \& Brannon, L. A. (2014). A test of the automaticity assumption of compliance tactics: Discouraging undergraduate binge drinking by appealing to consistency and reciprocity. Retrieved from http://krex.ksu.edu

\section{Published Version Information}

Citation: Conner, A. E., Miller, M. M., \& Brannon, L. A. (2014). A test of the automaticity assumption of compliance tactics: Discouraging undergraduate binge drinking by appealing to consistency and reciprocity. Communication Quarterly, 62(3), 269-284.

Copyright: (c) 2014 Eastern Communication Association

Digital Object Identifier (DOI): doi:10.1080/01463373.2014.911763

Publisher's Link:

http://www.tandfonline.com/doi/abs/10.1080/01463373.2014.911763\#.VDfaccVdVQw

This item was retrieved from the K-State Research Exchange (K-REx), the institutional repository of Kansas State University. K-REx is available at http://krex.ksu.edu 
A Test of the Automaticity Assumption of Compliance Tactics:

Discouraging Undergraduate Binge Drinking by Appealing to Consistency and Reciprocity 


\begin{abstract}
The mindfulness of compliance with requests making use of the commitment/consistency or the reciprocity principle was investigated. Participants $(N=129)$ received a foot-in-the-door (FITD) request (commitment/consistency application), a door-in-the-face (DITF) request (reciprocity application), or no request. Next, participants read either a weak or neutral message about the importance of moderate alcohol consumption then reported the likelihood of not drinking excessively for one week (target request). When accompanied by a weak message, the target request elicited less compliance if preceded by the DITF or FITD requests than by no initial request, suggesting compliance tactics sometimes increase thoughtfulness.
\end{abstract}

Keywords: Compliance, automaticity, binge drinking 


\section{A Test of the Automaticity Assumption of Compliance Tactics:}

Discouraging Undergraduate Binge Drinking by Appealing to Consistency and Reciprocity

Alcohol abuse is a serious health problem in the United States, resulting in an estimated 80,000 deaths each year, more than half of which are the result of binge drinking (CDC, 2012). Binge drinking is commonly operationalized as drinking five or more alcoholic beverages in a row for men, and four or more drinks in a row for women (Wechsler, Lee, Kuo, \& Lee, 2000). Based on this operationalization, binge drinking is most common among 18-24 year olds, and individuals in this age group report engaging in the most intense binge drinking (9.3 drinks per occasion) compared to other age groups (CDC, 2012). Binge drinking is especially prevelant among 18-24 year old college students, with approximately $40 \%$ of these young adults engaging in binge drinking (NIAAA, 2012b).

It is estimated that over 1,800 college students die from alcohol-related injuries, including motor vehicle accidents, and nearly 600,000 students suffer alcohol-related injuries each year (Hingson, Zha, \& Weitzman, 2009). College students who binge drink are more likely than those who do not binge drink to engage in a variety of dangerous behaviors, including driving after drinking (Wechsler et al., 2000). Additionally, binge drinkers are more likely to need medical attention as a result of overdosing on alcohol (Wechsler et al, 2000). Long-term binge drinking can have damaging effects on the liver, brain, heart, and pancreas and can increase the risk of developing cancer and contracting diseases as a result of weakening the immune system (NIAAA, 2012a). Less serious consequences of binge drinking include arguing with friends, forgetting behaviors in which they engaged while drinking, and missing classes and falling behind in schoolwork (Wechsler et al., 2000). 


\section{Applying Compliance Theory to Reducing Binge Drinking}

Despite the many interventions that have been implemented to reduce binge drinking, this behavior remains a major problem on college campuses (Wechsler et al., 2000). One goal of this paper is to apply compliance theory to the domain of reducing binge drinking behavior among college students. Specifically, this paper focuses on the application of two of the six primary principles of compliance: commitment/consistency and reciprocity (Cialdini \& Griskevicius, 2010).

\section{Commitment/Consistency}

The commitment/consistency rule is based on the long-held view that the need to present oneself as consistent is a strong motivator of behavior (e.g., Festinger, 1957; Heider, 1958). Thus, it is expected that people will be especially likely to comply with requests that are consistent with commitments they have made previously (Cialdini \& Griskevicius, 2010). The specific commitment/consistency technique investigated in the present research was the foot-inthe-door (FITD) tactic introduced by Freedman and Fraser (1966). In the FITD paradigm, participants are first asked to agree to a small request; typically the request is so small that almost everyone agrees to it. The second step in the FITD procedure is to ask participants to agree to a second, larger request. This request is actually the target request, and it is usually related in some way to the first. The FITD procedure is so named because "Like the proverbial salesperson who sticks a foot in the open door, getting the participant to agree with the easy request paves the way for agreement with the real request” (Burger, 1999, p. 303).

Since Freedman and Fraser's original work on FITD, this technique has been applied to a variety of practical situations, including charitable causes (Pliner, Hart, Kohl, \& Saari, 1974; Schwarzwald, Bizman, \& Raz, 1983) and organ donation (Carducci, Deuser, Bauer, Large, \& 
Ramaekers, 1989; Girandola, 2002). FITD also has been applied effectively to the domain of changing health-related behaviors including smoking reduction (Joule, 1987), encouraging women to schedule gynecological exams (Dolin \& Booth-Butterfield, 1995), and reducing drinking and driving behavior (Taylor \& Booth-Butterfield, 1993).

\section{Reciprococation}

Reciprocity is the second compliance principle that was a focus of this study. Following the reciprocation rule, people will be more likely to comply with a request made by another person who previously provided a favor (Cialdini \& Griskevicius, 2010). It has been argued that there exists a strong social norm to give back to those who have given to us (Gouldner, 1960; Singer, Van Holwyk, \& Maher, 2000), and the tendency to uphold this social norm is what arouses us to comply with requests based on the reciprocity principle, perhaps without engaging in much thought about the request (Cialdini \& Griskevicius, 2010).

The specific reciprocation method investigated in the present research was the door-inthe face (DITF) tactic (so named because the proverbial door-to-door salesperson could expect to have a door slammed in his or her face after making an intentionally extreme first request). It is expected that people will be more likely to comply with a target request if it is preceded by an extreme request (a DITF request), to which it is expected most anyone would not comply, than if the target request were made alone (Cialdini \& Griskevicius, 2010). If we do not fulfill the initial request, we may feel indebted to the requester, increasing the likelihood of compliance with later requests. Also, if the initial request is followed by a less extreme request (which is actually the target request), we may feel that the requester has done us a favor and has even sacrificed something on his or her end, making us feel more compelled to comply with the target request than we would have if the target request had not been preceded by the DITF request. 
DITF has been used to increase compliance with requests in several applied domains. For example, DITF has been applied to increase the likelihood that participants would donate blood (Cialdini \& Ascani, 1976) and to make donations to nonprofit organizations (Abrahams \& Bell, 1994; Bell, Abrahams, Clark, \& Schlatter, 1996; and Schwarzwald, Raz, \& Zvibel, 1979). Finally, the DITF procedure has been applied to improve compliance rates with health requests. For example, Millar (2001) applied the DITF tactic to increase the likelihood that participants would track their diets, as maintaining a record of one's diet is a reminder to eat healthfully.

\section{Prior Test of Mindless Compliance with Requests}

Much of human behavior occurs in an automatic fashion, and an evolutionary perspective has commonly been taken to explain the automaticity of compliance (Cialdini \& Griskevicius, 2010). That is, if certain situations or stimuli typically require the same response, it is advantegous for humans to develop automatic responses to these stimuli (Gigerenzer \& Goldstein, 1996). Given this natural human tendency to develop automatic responses to various stimuli, it has been inferred from the results of some compliance research that as a result of our social learning experiences, some human behaviors, including compliance, are produced in an automatic, mindless manner (e.g., Cialdini \& Griskevicius, 2010; Langer, 1989; Zimmatore, 1983). For example, in one study, Langer, Blank, and Chanowitz (1978) found that people waiting in line at a copying machine were more likely to comply with another person's request to step ahead in line if the requester provided a reason for the request, with the reason preceeded by “because,” regardles of the reason provided. Langer et al. (1978) inferred that because we learn to associate the word "because" with reason or explanation, and because we have a tendency to be more accepting of a request when provided with a reason for the request, people tend to automatically comply with requests that include "because.” 
While the foundational explanations for the effectiveness of compliance principles imply that people comply with these types of requests in a mindless or automatic manner, this assumption has been the focus of very few investigations. However, one study contested the automatic compliance theory with regard to another compliance principle, scarcity (Brannon \& Brock, 2001). In an applied context, Brannon and Brock (2001) presented restaurant customers with an opportunity to purchase a product, but manipulated the amount of time they had to take advantage of the opportunity: today only (high scarcity) or this year (low scarcity). To test the automatic compliance assumption, Brannon and Brock (2001) also included either a strong or weak argument for purchasing the product to investigate the extent to which participants were considering the merits of the offer (see Petty \& Wegener, 1998, for a more detailed explanation of assessing the roles of persuasion variables).

According to Brannon and Brock (2001), if participants were not differentiating between the strong and weak arguments because of high scarcity, then they would purchase the product regardless of the quality of the argument to purchase it. On the other hand, if participants were differentiating between the strong and weak arguments in the high scarcity condition, then they would differentiate in terms of their purchasing behavior: weak argument participants would not purchase the product; strong argument participants would purchase the product.

In fact, Brannon and Brock (2001) found that participants in the high scarcity conditions differentiated between the strong versus weak arguments more than did low scarcity participants. In other words, high scarcity seemed to increase message scrutiny. The consequence of Brannon and Brock’s (2001) findings is that weak messages seem less compelling than they would in the absence of the compliance variable, leading to a boomerang effect-a rejection of the offer. Contrary to the mindless responding that has been suggested by previous compliance research, 
these results were consistent with the increased thought hypothesis, given that "If a variable enhances the extent of thinking then one should see a greater polarization of attitudes in response to strong and weak arguments when the variable is present than when it is absent” (Petty, Fleming, \& White, 1999, p. 20).

One finding from the Brannon and Brock (2001) study in need of further investigation is that of the strong message condition. Participants in the high scarcity, strong argument condition complied the most with the request. Unfortunately, it is not possible to infer whether these participants complied automatically as a result of a scarcity cue effect, or whether they complied because they elaborated on the strong message. A neutral message may be a more appropriate comparison to a weak message. If Brannon and Brock (2001) had used a neutral message and found the same results as they did with the strong message, such results would indicate that compliance was the result of a cue effect. However, if they had found a moderate level of compliance with the neutral message, the results would be an indication that the compliance tactic did not serve as a cue, but that participants were thinking about the message.

\section{Automatic Versus Thoughtful Compliance}

One purpose of the present research was to investigate the issue of mindless compliance with requests, focusing specifically on compliance with the sequential request tactics of foot-inthe-door (FITD) and door-in-the-face (DITF). These tactics have not been as widely researched as other compliance principles; especially with regard to why they work in gaining compliance. Burger (1999) wrote, “Questions remain about the effectiveness of the FITD procedure, the conditions under which it will be found, and how to explain successful demonstrations of the effect” (p. 304). Similarly, O’Keefe and Hale (1998) concluded, “There is substantial variability in DITF effects, even under optimal conditions” (p. 27). Perhaps this variability can be attributed 
to the as yet untested assumption that compliance with the second (target) request within the DITF and FITD paradigms sometimes occurs mindlessly following a response to the initial request. The notion of automatic compliance to sequential-request techniques may be accurate in some situations, but there may be some situations in which the use of these tactics elicit increased thought, leading to differential responding to target requests.

\section{Overview of Research}

This research investigated whether compliance with target requests within the sequential request paradigm (FITD and DITF requests) is automatic within the context of reducing binge drinking among college students. It was predicted that, consistent with the increased thought hypothesis (Petty et al., 1999), FITD and DITF participants would agree differentially to the target request as a function of message strength, indicating that use of these tactics do not always result in mindless compliance.

\section{Method}

\section{Pretest}

Prior to conducting the main experiment, participants rated either a neutral $(N=26)$ or weak $(N=27)$ argument in terms of persuasiveness (along a 7-point scale, where $1=$ not at all persuasive and 7 = extremely persuasive) in a between-participants design. Both messages were 267 words long. However, the neutral message described negative consequences of binge drinking in a factual manner (e.g., "students who frequently drink excessive amounts of alcohol are 6 to 17 times more likely than students who do not drink excessively to miss class, get behind in school work, engage in unplanned sex, get in trouble with police, damage property, or get hurt or injured”). Although these events are more likely for people who are frequent, heavy drinkers, they are still not common for most college students (getting in trouble with the police, damaging 
property, being injured). This would reduce the personal relevance (and hence the persuasiveness) of the appeals for the average drinker. The weak message discussed consequences of binge drinking in a very opinion-based way (e.g., "people say they drink to have fun, but people our parents' age had plenty of fun without drinking themselves silly. If it was good enough for them, it's good enough for us...And everyone knows that drinking too much alcohol makes people feel 'buzzed' or 'tipsy.' Who really wants to feel like that?").

The average persuasiveness rating of the neutral message was at the midpoint of the scale ( $M=3.88, S D=1.24$ ) and was significantly higher than the average persuasiveness rating of the weak message $(M=2.85, S D=1.41), t(51)=2.83, p<.01$.

In order to determine the most appropriate items for the FITD, DITF, and target requests, participants rated how likely they were to perform a variety of tasks related to curbing their drinking behavior. In total, participants rated 21 items along a 7-point scale, where $1=$ extremely unlikely and $7=$ extremely likely.

Logically, pretest items with a relatively high likelihood rating would serve well as FITD initial items, while items with a relatively low likelihood rating would serve well as DITF initial items. Pretest items rated in the middle of the scale would serve well as target items. Consistent with this logic, the selected FITD item (not drinking for one night in the next week) received a mean likelihood rating of $5.35(N=62, S D=2.08)$; the selected DITF item (not drinking at all in the next month, with name being published in the school newspaper) received a mean rating of $3.58(N=62, S D=2.46)$; and the target item (not drinking excessively for one week) received a mean likelihood rating of $4.89(N=61, S D=1.87)$.

\section{Main Experiment}

Participants. A prerequisite for participation in the study was that students drink alcohol 
at least occasionally. Participants were recruited from introductory and upper-level psychology and marketing classes, and received credit for participating $(N=160)$. Consistent with the methods employed by sequential request researchers, the data of participants who did not agree to help with the first request in the FITD manipulation $(n=4)$, and participants who did agree to help with the first request in the DITF manipulation $(n=15)$ were not included in the analysis. Also, 12 participants provided incomplete responses, and their data were not included in the analysis, resulting in a final sample of 129 participants. Participants $(N=129)$ completed a lengthy demographic questionnaire. They reported their age $(M=20.40$ years, $S D=1.87)$, gender (55.3\% were female), ethnicity (93.5\% were White, $0.7 \%$ were Black, $1.4 \%$ were Hispanic, and 4.3\% reported other ethnicities), and year in school (39.7\% were freshmen, 17.0\% were sophomores, $14.9 \%$ were juniors, and 28.4\% were seniors). Embedded in this questionnaire, participants also reported the number of drinks they consume per drinking occasion $(M=6.25$ drinks, $S D=3.61)$.

Materials and Procedure. At the beginning of the experiment, students were told that they would be asked to help establish a new responsible drinking group on campus, and that their choice to help the group was completely voluntary. After providing an overview of the experimental tasks, the researcher randomly assigned participants to conditions when distributing a packet of materials. In all conditions, the packet participants received contained a cover sheet describing the responsible drinking group, called Responsible Alcohol Consumption Team, or ReACT. The group was described as containing researchers and students who realize that many students consider drinking an important part of college life, and one that would like to see students drink more responsibly when they consume alcohol.

For the participants in the sequential-request conditions, the second page of the packet 
asked for compliance with an initial request. To assess compliance with the FITD request, participants were presented with the statement, "I acknowledge, by signing below, that I will not drink alcohol for one night in the next week. This is any one night that I choose in the next week" and those who signed the form were considered to have complied with the request. Compliance with the DITF request was measured by whether or not students signed their names in response to the statement, "I acknowledge, by signing below, that I will not drink alcohol at all for the entire month, effective immediately. I also agree to having my name published in the Collegian.” Finally, consistent with standard sequential-request research procedures, control participants were not asked to agree to an initial request.

After participants were asked to agree to the initial request (or not in the control condition), they read the neutral or weak message about the dangers of excessive drinking as an example of the sort of information the group planned to distribute on the students' campus. Participants in all conditions were then asked to agree to the target request, to not drink excessive amounts of alcohol for one week. The main dependent variable was the response to the question "What is the likelihood that you would not drink to excess for one week? (Write a number from 0 to 100 on the line. 0 means you are not at all likely to follow through, and 100 means you are completely likely to follow through).” In this initial test of the automaticity assumption underlying the effectiveness of appeals to reciprocity and consistency, a behavioral intention measure was used as the dependent variable because most models of health behavior consider some form of behavioral intention as being necessary (and assuming there are no barriers to performance of the behavior, sufficient) for producing a behavior (Fishbein, Triandis, Kanfer, Becker, Middlestadt, \& Eichler, 2001). More generally, past research has demonstrated that behavioral intentions are a good predictor of behavior, especially when there is a clear timeframe 
specified for the behavior (Ajzen, 1988; Ajzen \& Fishbein, 1980).

As noted in the description of participants above, participants reported demographic information (gender, age, ethnicity, and year in school) and alcohol consumption. Specifically, for alcohol consumption, participants were asked, "On average, how many alcoholic beverages do you consume each time you drink? (NOTE: One drink = one bottle of beer, one glass of wine, one wine cooler, or one shot of liquor).” Finally, participants completed a 10-item version of the Marlowe-Crowne Social Desirability Scale (the M-C 2(10); Strahan \& Gerbasi, 1972), which measures the tendency of some research participants to present themselves in a favorable, socially desirable manner. Participants indicate whether ten statements are "true” or "false" of them (e.g., "I never hesitate to go out of my way to help someone in trouble" "I sometimes feel resentful when I don't get my way”). For each item, participants receive one point if they give the more socially desirable response and no points for the less socially desirable response. The higher a participant's total score for the measure, the greater is his or her tendency to respond in a socially desirable manner. The tendency to appear socially desirable can affect the responses provided by research participants when asked to report their behavior, to the extent that respondents may provide inaccurate responses in order to manage the impression others have of them (Paulhus, 1991).

\section{Results}

The dependent variable was the estimated likelihood of not drinking to excess for one week. Refer to Table 1 for the mean likelihood of following through on the target request as a function of condition (note that all means reported are adjusted for the covariates: average number of drinks per occasion and social desirability).

The data were submitted to a 2(Strength: weak/neutral) $\times 3$ (Appeal: DITF/FITD/Control) 
$\times$ 2(Gender: male/female) Analysis of Covariance (ANCOVA) with number of drinks per occasion and level of social desirability as covariates. The drinks per occasion covariate was included because a person's general tendency to drink a certain amount of alcohol could influence that person's willingness to agree to drink responsibly; while the social desirability covariate was included because a person's level of social desirability has been shown to affect how likely it is a person will respond honestly when self-reporting attitudes and behaviors (Paulhus, 1991). ${ }^{1}$

The analysis revealed a significant Strength $\mathrm{x}$ Appeal disordinal interaction, $F(2,115)=$ 3.00, $p=.05, \eta^{2}=.05$. Planned comparisons were carried out to investigate this significant interaction. Consistent with the increased thought hypothesis, the DITF appeal accompanied by the weak message yielded significantly lower likelihood ratings $(M=66.91 \%, S E=5.75)$ of following through on the target request than the control appeal (no initial request) accompanied by the weak message $(M=82.22 \%, S E=4.99), F(1,115)=4.12, p=.04, \eta^{2}=.09$. Additionally, the FITD appeal accompanied by the weak messaged yielded significantly lower likelihood ratings of following through on the target request $(M=67.22 \%, S E=4.81)$ than the control appeal (no initial request) combined with the weak message $(M=82.22 \%, S E=4.99), F(1,115)$ $=4.62, p=.03, \eta^{2}=.09$.

Further, planned comparisons were carried out to investigate the effects of argument strength within appeal. Within the FITD appeal, the weak message yielded significantly lower likelihood ratings $(M=67.22 \%, S E=4.81)$ of following through on the target request than the neutral message $(M=83.34 \%, S E=5.44), F(1,115)=4.93, p=.03, \eta^{2}=.10$. Within the DITF appeal, the weak message yielded marginally lower likelihood ratings $(M=66.91 \%, S E=5.75)$ of following through on the target request than the neutral message ( $M=83.75 \%, S E=7.14$ ), 
$F(1,115)=3.31, p=.07, \eta^{2}=.09$. However, within the control conditions (no initial request), there was no significant difference between the likelihood ratings for the neutral message $(M=$ $76.60 \%, S E=4.94)$ and the weak message $(M=82.22 \%, S E=4.99), F(1,115)=0.64, p=.42$, $\eta^{2}=.00$. The main effect of Message Strength was significant but was qualified by the significant Strength x Appeal interaction. No other effects were significant.

\section{Discussion}

The present research investigated whether the sequential request tactics FITD and DITF may be useful tools for encouraging responsible drinking among college students. The study also examined the extent to which individuals mindlessly comply with requests when these tactics are used. The results supported the hypothesis that compliance with requests that apply the principles of reciprocity and commitment/consistency, such as the DITF and FITD tactics, may not always result in automatic and mindless compliance as has been previously suggested (Cialdini \& Griskevicius, 2010). In the current study, when a weak message was presented after either a DITF or FITD request and before the target request, likelihood of complying with the target request was significantly lower than if an initial request did not precede the target request (effect sizes were moderate, both $\eta^{2}=.09$ ). Further, in the FITD condition, compliance likelihood was significantly lower when a weak message was presented than when a neutral message was presented (effect size was moderate, $\eta^{2}=.09$ ). The same pattern of results was found in the DITF condition, but the compliance likelihood difference between weak and neutral message conditions was marginally significant $(p=.07)$. However, compliance likelihood did not differ between the message conditions when no initial request was presented (control condition).

\section{Contribution of the Current Research to the Literature}

Although our data demonstrated that there may be conditions under which the use of 
sequential request tactics are associated with reduced compliance, the data suggest neither that such conditions will have a devastating effect on compliance nor that these tactics are never effective. In fact, with average likelihood of compliance rates greater than $50 \%$ in both the FITD and DITF conditions (regardless of message strength), our data suggest that there may be a benefit to incorporating these tactics into responsible drinking campaigns among college students. These results are consistent with other research in the domain of health communication that has found compliance tactics such as FITD and DITF to be effective at promoting positive health-related change. For example, in a field study, women were more likely to schedule a gynecological exam (target request) if they had previously complied with a request to accept a breast self-examination demonstration card (FITD request) than if this request had not be presented to them (Dolin \& Booth-Butterfield, 1995).

Still, the fact that the current study is consistent with previous research identifying moderators of compliance tactic efficacy should not be ignored. Brannon and Brock (2001) also examined the moderating effect of message strength on compliance but they focused on requests involving time restriction (an application of the scarcity principle). Under conditions of high time restriction, compliance rates were differentiated by message strength such that compliance was significantly lower if high time restriction was paired with a weak message than if paired with a strong message. Also, as in the current study, Brannon and Brock (2001) found that compliance rates did not differ between message strength conditions when a tactic based on scarcity principle was absent (only the informational message and the target request were presented). In other words, in both the current research and that conducted by Brannon and Brock (2001), pairing a weak message with the use of a compliance tactic seemed to have a negative, though not necessarily detrimental, impact on compliance. 
Aside from examining different compliance tactics, the current study differed from Brannon and Brock’s (2001) research by comparing weak and neutral messages rather than weak and strong messages. When compliance rates are high in the strong message condition, it is not possible to differentiate whether compliance is due to a cue/automaticity effect produced by the compliance tactic or a message processing effect elicited in response to the strong message (see Petty \& Cacioppo, 1986). If the automaticity assumption is correct and use of tactics such as the DITF or FITD techniques produce mindless compliance, then when exposed to one of these tactics and presented with a neutral message, we should expect participants to report higher levels of compliance than those who are presented with a neutral message but not exposed to a compliance tactic (our control condition). However, in our study, among participants presented with the neutral message, compliance likelihood reports did not differ significantly among the DITF, FITD, and no compliance tactic (control) condition. Thus, the lack of a significant difference between either of the tactic conditions and the control condition suggests that the application of compliance principles may not always result in mindless compliance.

Research also has demonstrated how trait or individual difference variables can influence the effectiveness of sequential request tactics (Burger, \& Guadagno, 2003; Guadagno, Asher, Demaine, \& Cialdini, 2001; Kassner \& Graziano, 2012). For example, Burger and Guadagno (2003) found that individuals with high self-concept clarity were more likely than those with low self-concept clarity to comply with a target request (volunteering their time to help with a cause) if they previously complied with a related request (signing a letter supporting the cause) than if they were not presented with the earlier request. Burger and Guadagno’s (2003) work supports the self-perception explanation for why the FITD technique increases compliance, but their work also indicates that this technique may not be effective at inducing compliance equally across 
individuals. Certain individuals (e.g., those high in self-concept clarity) may be more likely than others (e.g., those low in self-concept clarity) to adjust their self-concept as a result of engaging in a behavior and feel motivated to be consistent by also engaging in other related behaviors (Burger \& Guadagno, 2003; Guadagno et al., 2001).

Also, the current study is only one of a few that has examined moderators of sequential request efficacy in a health behavior context. For example, Millar (2001) found that adults were more likely to agree to keeping a detailed record of their meals over a four day period and mailing the record back to the experimenter if they were previously asked to keep a detailed record of their meals for one month (DITF) than if this more extreme request were not made. However, these results were found among participants who were told that keeping the record would be beneficial either to the participant or to a third party health organization. If told that the meals record would be used to help the requester with his research, the DITF request backfired, and participants actually were more likely to comply with the target request if the initial extreme request were not presented.

\section{Health Behavior Models and Compliance Tactic Efficacy}

A consideration of health behavior theories and models might help explain why sequential request tactics can be effective at influencing health behaviors. The health belief model identifies perceived barriers as an important factor influencing whether or not people will engage in health behavior change (Rosenstock, Strecher, \& Becker, 1988). Also, the health belief model and social cognitive theory (Bandura, 1977) both acknowledge the role that self-efficacy plays in the likelihood that people will be persuaded by a health campaign to change their behavior - if they do not believe they can effectively perform the target behavior, they are less likely to do so. Consistent with the popular self-perception explanation for the effectiveness of 
the FITD technique, perhaps compliance with the initial small request arouses the necessary shift in perceived self-efficacy that the health belief model and social cognitive theory suggest is needed for behavior change. That is, if individuals successfully engage in the initial behavior, they may infer that they will be successful at engaging in other related behaviors, and their perceived self-efficacy for the target behavior is greater than it would have been without engaging in the initial behavior. The transtheoretical model (Prochaska \& DiClemente, 1983) also might be important to consider in attempts to understand the efficacy of sequential request compliance procedures (particularly the FITD tactic) in health campaigns. That is, if an individual is in the precontemplation or contemplation stage of behavior change, it is possible that getting an individual to comply with a small request can help nudge them into the next stage of change as a result of modifying their self-perception.

\section{Future Directions}

Finally, we provide several ideas for extending research on the application of compliance principles on a broad level as well as specifically related to applications in health campaigns. The present study did not allow participants to perform the initial request before they were asked to comply with the target request, so future research might examine performance of the initial request. Freedman and Fraser (1966) noted that allowing request recipients to perform the initial FITD request increases compliance with the target request, relative to not actually performing it. In such situations, one might even expect stronger effects. Similarly, it should be noted that although behavioral intentions, as measured in the present study, are frequently good predictors of subsequent behavior (Ajzen \& Fishbein, 1980; Ajzen, 1988; Fishbein et al. 2001), future research should follow up on the behavioral intentions and measure actual drinking behavior in response to the target request. It also is important to determine the extent to which the sequential 
request compliance tactics lose some of their impact when made on paper as compared to faceto-face interactions, which are more time-consuming and costly to implement. Extending this idea even further, another interesting avenue for future research would be to examine whether sequential requests like the FITD and DITF can be used effectively over the internet, through the use of “cookies.” The ability to tailor messages over the internet has been effectively used to promote other types of health messages (Rimal \& Adkins, 2003).

Future research on the use of sequential request tactics in health campaigns might examine whether use of one of these tactics is more is more appropriate than the other, depending on the target health behavior. The DITF tactic has been found to be more effective with shorter delays between the initial and target request, but the delay time between requests does not seem to matter for the FITD tactic (Dillard, Hunter, \& Burgoon, 1984). For certain behaviors and in certain contexts, it may be feasible and sensible to have a short delay between requests, and in such cases, either the FITD or DITF tactic could be employed. However, if the behavior and/or context require a delay between requests, then the FITD tactic should be used. In the current study, the delay between requests was short, so it was feasible to test both FITD and DITF tactics. One study found the FITD technique with a relatively long delay period to be effective at encouraging taxi use among alcohol-intoxicated bar-goers (Taylor \& BoothButterfield, 1993). Future research might examine whether the FITD technique with a longer delay period than that used in the current study can be effective at encouraging responsible alcohol consumption among college students.

\section{Conclusion}

The present research is consistent with previous studies demonstrating on a broad level that health campaigns can benefit from the use of techniques such as the FITD and DITF tactics 
and specifically that these methods may be effective at encouraging responsible alcohol consumption among college students. However, the primary implication of the results from this study and others is that applying compliance principles under certain conditions, such as when a weak message accompanies a request, may actually increase thoughtfulness, reducing the likelihood of compliance with the target request. It is important to note that our data do not suggest that pairing a weak message with the FITD or DITF tactics will completely eliminate compliance with the target request, nor that these tactics are ineffective and should never be used in health campaigns. Still, when money, time, and human resources are invested in health campaign programs, it is important for campaign coordinators to be aware of factors that might have a negative impact on the effectiveness of the campaign. In terms of the implications for anti-binge drinking campaigns, if future research identifies a message containing very compelling arguments, and the use of tactics such as FITD or DITF increases the message recipients' scrutiny of this compelling message, then the potential exists for creating an effective anti-binge drinking social marketing campaign targeting college students. On the other hand, in situations where the campaign cannot afford to adequately test its message and ensure that it is compelling, the present research suggests that the campaign should avoid the use of the FITD or DITF tactics, and only make the target request. It is important that research continue to investigate the extent to which compliance is mindless or thoughtful with regard to these and other compliance tactics. Knowing how these tactics work to gain compliance is important because this will help predict when they should be used. 


\section{References}

Abrahams, M. F., \& Bell, R. A. (1994). Encouraging charitable contributions: An examination of three models of door-in-the-face compliance. Communication Research, 21, 131-153.

Ajzen, I. (1988). Attitudes, personality, and behavior. Dorsey Press: Chicago.

Ajzen, I., \& Fishbein, M. (1980). Understanding attitudes and predicting social behavior. Englewood Cliffs, NJ: Prentice-Hall.

Bandura, A. (1977). Self-efficacy: Toward a unifying theory of behavioral change. Psychologicla Review, 84, 191-215.

Bell, R. A., Abrahams, M. F., Clark, C. L., \& Schlatter, C. (1996). The door-in-the-face compliance strategy: An individual differences analysis of two models in an AIDS fundraising context. Communication Quarterly, 44, 107-124.

Brannon, L. A., \& Brock, T. C. (2001). Limiting time for responding enhances behavior corresponding to the merits of compliance principles: Refutations of heuristic-cue theory in service and consumer settings. Journal of Consumer Psychology, 10, 135-146.

Burger, J. M. (1999). The foot-in-the-door compliance procedure: A multiple-process analysis and review. Personality and Social Psychology Review, 3, 303-325.

Burger, J. M., \& Guadagno, R. E. (2003). Self-concept clarity and the foot-in-the-door procedure. Basic and Applied Social Psychology, 25, 79-86.

Carducci, B. J., Deuser, P. S., Bauer, A., Large, M., \& Ramaekers, M. (1989). An application of the foot in the door technique to organ donation. Journal of Business and Psychology, 4, 245-249.

Centers for Disease Control and Prevention. (2012). Vital signs: Binge drinking prevalence, frequency, and intensity among adults-United States, 2010. Morbidity and Mortality 
Weekly Report, 61, 14-19.

Cialdini, R. B., \& Ascani, K. (1976). Test of a concession procedure for inducing verbal, behavioral, and further compliance with a request to give blood. Journal of Applied Psychology, 61, 295-300.

Cialdini, R. B., \& Griskevicius, V. (2010). Social influence. In R. F. Baumeister, \& E. J. Finkel (Eds.), Advanced social psychology: The state of the science (pp. 385-417). Oxford University Press: Oxford, NY.

Dillard, J. P., Hunter, J. E., \& Burgoon, M. (1984). Sequential request persuasive strategies: Meta-analysis of foot-in-the-door and door-in-the-face. Human Communication Research, 10, 461-488.

Dolin, D. J., \& Booth-Butterfield, S. (1995). Foot-in-the-door and cancer prevention. Health Communication, 7, 55-66.

Festinger, L. (1957). A theory of cognitive dissonance. Stanford: Stanford University Press.

Fishbein, M., Triandis, H. C., Kanfer, F. H., Becker, M., Middlestadt, S. E., \& Eichler, A. (2001). Factors influencing behavior and behavior change. Handbook of health psychology (pp.3-17). Lawrence Erlbaum Associates: Mahwah, NJ.

Freedman, J. L. \& Fraser, S. C. (1966). Compliance without pressure: The foot-in-the-door technique. Journal of Personality and Social Psychology, 4, 195-202.

Gigerenzer, G., \& Goldstein, D. G. (1996). Reasoning the fast and frugal way: Models of bouned rationality. Psychological Review, 103, 650-669.

Girandola, F. (2002). Sequential requests and organ donation. The Journal of Social Psychology, 142, 171-178.

Gouldner, A. W. (1960). The norm of reciprocity: A preliminary statement. American 
Sociological Review, 25, 161-178.

Guadagno, R. E., Asher, T., Demaine, L. J., \& Cialdini, R. B. (2001). When saying yes leads to saying no: Preference for consistency and the reverse foot-in-the-door effect. Personality and Social Psychology Bulliten, 27, 859-867.

Heider, F. (1958). The psychology of interpersonal relations. New York: Wiley

Hingson, R. W., Zha, W., \& Weitzman, E. R. (2009). Magnitude of and trends in alcohol-related mortality and morbidity among U.S. college students ages 18-24, 1998-2005. Journal of Studies on Alcohol and Drugs Supplement, 16, 12-20.

Joule, R. V. (1987). Tobacco deprivation: The foot-in-the-door technique versus the low-ball technique. European Journal of Social Psychology, 17, 361-365.

Kassner, M. P., \& Graziano, W. G. (2012, January). Don't think about it: Cognitive processing increases susceptibility to the door-in-the-face compliance tactic. Poster session presented at the meeting of the Society for Personality and Social Psychology, San Diego, CA.

Langer, E. J. (1989). Minding matters. In L. Berkowitz (Ed.), Advances in experimental social psychology (Vol. 22). New York: Academic Press.

Langer, E., Blank, A., \& Chanowitz, B. (1978). The mindlessness of ostensibly thoughtful action: The role of "placebic" information in interpersonal interaction. Journal of Personality and Social Psychology, 36, 635-642.

Millar, M. G. (2001). Promoting health behaviours with door-in-the-face: The influence of the beneficiary of the request. Psychology, Health \& Medicine, 6, 115-119.

National Institute on Alcohol Abuse and Alcoholism (2012a). Beyond hangovers: Understanding alcohol's impact on your health (NIH Publication No. 10-7604). Retrieved from 
http://pubs.niaaa.nih.gov/publications/Hangovers/beyondHangovers.pdf

National Institute on Alcohol Abuse and Alcoholism (2012b). College drinking. Retrieved from http://pubs.niaaa.nih.gov/publications/CollegeFactSheet/CollegeFactSheet.pdf

O’Keefe, D. J., \& Hale, S. L. (1998). The door-in-the-face influence strategy: A random-effects meta-analytic review. In M. E. Roloff (Ed.), Communication yearbook 21, (pp. 1-33). Thousand Oaks, CA: Sage.

Paulhus, D. L. (1991). Measurement and control of response bias. In J. P. Robinson. P. P. Shaver, \& L. S. Wrightsman (Eds.), Measures of personality and social psychological attitudes (pp.17-59). New York: Academic Press.

Petty, R. E., \& Cacioppo, J. T. (1986). The Elaboration Likelihood Model of persuasion. In L. Berkowitz (Ed.), Advances in experimental social psychology (Vol. 19, pp. 123-205). New York: Academic Press.

Petty, R. E., Fleming, M. A., \& White, P. H. (1999). Stigmatized sources and persuasion: Prejudice as a determinant of argument scrutiny. Journal of Personality and Social Psychology, 76, 19-34.

Petty, R. E., \& Wegener, D. T. (1998). Attitude change: Multiple roles for persuasion variables. In D. Gilbert, S. Fiske, \& G. Lindzey (Eds.), Handbook of social psychology (4 ${ }^{\text {th }}$ ed., pp. 323-390). New York: McGraw Hill.

Pliner, P., Hart, H., Kohl, J., \& Saari, D. (1974). Compliance without pressure: Some further data on the foot-in-the-door compliance technique. Journal of Experimental Social Psychology, 10,17-22.

Prochaska, J., \& DiClemente, C. C. (1983). Stages and process of self-change of smoking: Toward an integrative model of change.” Journal of Consulting and Clinical Psychology, 


\section{1, 390-395.}

Rimal, R. N., \& Adkins, A. D. (2003). Using computers to narrowcast health messages: The role of audience segmentation, targeting, and tailoring in health promotion. In T. L. Thompson, A. M. Dorsey, I. Katherine, \& R. Parrott. (Eds.), Handbook of health communication (pp. 497-513). Mahwah, NJ: Lawrence Erlbaum Associates.

Rosenstock, I. M., Strecher, V. J., \& Becker, M. H. (1988). Social learning theory and the health belief model. Health Education Quarterly, 15, 175-183.

Schwarzwald, J., Bizman, A., \& Raz, M. (1983). The foot-in-the-door paradigm: Effects of second request size on donation probability and donor generosity. Personality and Social Psychology Bulletin, 9, 443-450.

Schwarzwald, J., Raz, M., \& Zvibel, M. (1979). The applicability of the door-in-the-face technique when established behavioral customs exist. Journal of Applied Social Psychology, 9, 576-586.

Singer, E., Van Holwyk, J., \& Maher, M. P. (2000). Experiments with incentives in telephone surveys. Public Opinion Quarterly, 64, 171-188.

Strahan, R., \& Gerbasi, K. C. (1972). Short, homogenous versions of the Marlowe-Crowne Social Desirability Scale. Journal of Clinical Psychology, 28, 191-193.

Taylor, T., \& Booth-Butterfield, S. (1993). Getting a foot-in-the-door with drinking and driving: A field study of healthy influence. Communication Research Reports, 10, 95-101.

Wechsler, H., Lee, J. E., Kuo, M., \& Lee, H. (2000). College binge drinking in the 1990's: A continuing problem--Results of the Harvard School of Public Health 1999 College Alcohol Study. Journal of American College Health, 48, 199-210. 
Zimmatore, J. J. (1983). Consumer mindlessness: I believe it, but I don’t see it. Proceedings of the Division of Consumer Psychology, American Psychological Association Convention, Anaheim, CA. 


\section{Footnote}

${ }^{1}$ Prior to including baseline drinking and social desirability as covariates in the analysis, we ensured that the assumption of independence of the covariate and treatment effect and the assumption of homogeneity of regression slopes had been met. To test the assumption of independence, two ANOVAs were conducted (one for each covariate) to test for differences in the covariate across experimental groups. All effects were nonsignificant, indicating the assumption had been met. To test the assumption of homogeneity of regression slopes, we conducted a custom model of our ANCOVA, which included the interactions between the covariates and the independent variables. This analysis also did not produce significant effects, indicating the assumption was met. 
Table 1

Mean Likelihood of Not Drinking to Excess, Standard Error, and Cell Size as a Function of Message Strength and Appeal Type

\begin{tabular}{lccc}
\hline Appeal & \multicolumn{2}{c}{ Argument Strength } & Row Means \\
& Weak & Strong & \\
& $66.91^{\mathrm{A} 1}$ & $83.75^{\mathrm{A} 1}$ & 75.33 \\
DITF & $(5.75)$ & $(7.14)$ & $(4.54)$ \\
& 18 & 15 & 33 \\
& $67.22^{\mathrm{A} 1}$ & & 75.28 \\
FITD & $(4.81)$ & $83.34^{\mathrm{A} 2}$ & $(3.63)$ \\
& 26 & $(5.44)$ & 47 \\
& $82.22^{\mathrm{B} 1}$ & 21 & 79.41 \\
Control & $(4.99)$ & 76.60 & $(3.52)$ \\
& 24 & $(4.94)$ & 49 \\
& & 25 & 76.67 \\
Column Means & 72.11 & 81.23 & $(2.27)$ \\
& $(3.00)$ & $(3.42)$ & 129 \\
\hline
\end{tabular}

Note. Means are adjusted for the covariates (number of alcoholic beverages consumed per occasion and social desirability). Standard errors (in parentheses) and $n$ s (in italics) appear below means. Cell means in the same column that do not share alphabetical superscripts differ at the $p$ $<.05$ level, and cell means in the same row that do not share the same numerical superscripts differ at the $p<.05$ level, according to planned comparisons carried out to investigate the significant Strength $\times$ Appeal interaction. 